\title{
Additive functional inequalities in Banach spaces
}

\author{
Gang Lu' and Choonkil Park²*
}

\author{
"Correspondence: \\ baak@hanyang.ac.kr \\ 2Department of Mathematics, \\ Research Institute for Natural \\ Sciences, Hanyang University, Seoul, \\ 133-791, Korea \\ Full list of author information is \\ available at the end of the article
}

\section{Abstract}

In this paper, we prove the Hyers-Ulam stability of the following function inequalities:

$$
\begin{array}{ll}
\|f(x)+f(y)+f(z)\| \leq\left\|K f\left(\frac{x+y+z}{K}\right)\right\| \quad(0<|K|<3), \\
\|f(x)+f(y)+K f(z)\| \leq\left\|K f\left(\frac{x+y}{K}+z\right)\right\| \quad(0<K \neq 2)
\end{array}
$$

in Banach spaces.

MSC: Primary 39B62; 39B52; 46B25

Keywords: Hyers-Ulam stability; additive functional inequality; additive mapping

\section{Introduction and preliminaries}

The stability problem of functional equations originated from the question of Ulam [1] in 1940 concerning the stability of group homomorphisms. Let $\left(G_{1}, \cdot\right)$ be a group and let $\left(G_{2}, *\right)$ be a metric group with the metric $d(\cdot, \cdot)$. Given $\epsilon>0$, does there exist a $\delta 0$ such that if a mapping $h: G_{1} \rightarrow G_{2}$ satisfies the inequality $d(h(x \cdot y), h(x) * h(y))<\delta$ for all $x, y \in G_{1}$, then there exists a homomorphism $H: G_{1} \rightarrow G_{2}$ with $d(h(x), H(x))<\epsilon$ for all $x \in G_{1}$ ? In other words, under what condition does there exist a homomorphism near an approximate homomorphism? The concept of stability for a functional equation arises when we replace the functional equation by an inequality which acts as a perturbation of the equation. In 1941, Hyers [2] gave the first affirmative answer to the question of Ulam for Banach spaces. Let $f: E \rightarrow E^{\prime}$ be a mapping between Banach spaces such that

$$
\|f(x+y)-f(x)-f(y)\| \leq \delta
$$

for all $x, y \in E$ and for some $\delta>0$. Then there exists a unique additive mapping $T: E \rightarrow E^{\prime}$ such that

$$
\|f(x)-T(x)\| \leq \delta
$$

for all $x \in E$. Moreover, if $f(t x)$ is continuous in $t \in \mathbb{R}$ for each fixed $x \in E$, then $T$ is $\mathbb{R}$ linear. In 1978, Th.M. Rassias [3] proved the following theorem.

(c) 2012 Lu and Park; licensee Springer. This is an Open Access article distributed under the terms of the Creative Commons Attribution License (http://creativecommons.org/licenses/by/2.0), which permits unrestricted use, distribution, and reproduction in any medium, provided the original work is properly cited. 
Theorem 1.1 Let $f: E \rightarrow E^{\prime}$ be a mapping from a normed vector space $E$ into a Banach space $E^{\prime}$ subject to the inequality

$$
\|f(x+y)-f(x)-f(y)\| \leq \epsilon\left(\|x\|^{p}+\|y\|^{p}\right)
$$

for all $x, y \in E$, where $\epsilon$ and $p$ are constants with $\epsilon>0$ and $p<1$. Then there exists a unique additive mapping $T: E \rightarrow E^{\prime}$ such that

$$
\|f(x)-T(x)\| \leq \frac{2 \epsilon}{2-2^{p}}\|x\|^{p}
$$

for all $x \in E$. If $p<0$, then inequality (1.1) holds for all $x, y \neq 0$, and (1.2) for $x \neq 0$. Also, if the function $t \mapsto f(t x)$ from $\mathbb{R}$ into $E^{\prime}$ is continuous in $t \in \mathbb{R}$ for each fixed $x \in E$, then $T$ is $\mathbb{R}$-linear.

In 1991, Gajda [4] answered the question for the case $p>1$, which was raised by Th.M. Rassias. On the other hand, J.M. Rassias [5] generalized the Hyers-Ulam stability result by presenting a weaker condition controlled by a product of different powers of norms.

Theorem 1.2 [6, 7] If it is assumed that there exist constants $\Theta \geq 0$ and $p_{1}, p_{2} \in \mathbb{R}$ such that $p=p_{1}+p_{2} \neq 1$, and $f: E \rightarrow E^{\prime}$ is a mapping from a norm space $E$ into a Banach space $E^{\prime}$ such that the inequality

$$
\|f(x+y)-f(x)-f(y)\| \leq \epsilon\|x\|^{p_{1}}\|y\|^{p_{2}}
$$

holds for all $x, y \in E$, then there exists a unique additive mapping $T: E \rightarrow E^{\prime}$ such that

$$
\|f(x)-T(x)\| \leq \frac{\Theta}{2-2^{p}}\|x\|^{p}
$$

for all $x \in E$. If, in addition, for every $x \in E, f(t x)$ is continuous in $t \in \mathbb{R}$ for each fixed $x \in E$, then $T$ is $\mathbb{R}$-linear.

More generalizations and applications of the Hyers-Ulam stability to a number of functional equations and mappings can be found in [8-11].

In [12], Park et al. investigated the following inequalities:

$$
\begin{aligned}
& \|f(x)+f(y)+f(z)\| \leq\left\|2 f\left(\frac{x+y+z}{2}\right)\right\|, \\
& \|f(x)+f(y)+f(z)\| \leq\|f(x+y+z)\|, \\
& \|f(x)+f(y)+2 f(z)\| \leq\left\|2 f\left(\frac{x+y}{2}+z\right)\right\|
\end{aligned}
$$

in Banach spaces. Recently, Cho et al. [13] investigated the following functional inequality:

$$
\|f(x)+f(y)+f(z)\| \leq\left\|K f\left(\frac{x+y+z}{K}\right)\right\| \quad(0<|K|<|3|)
$$


in non-Archimedean Banach spaces. Lu and Park [14] investigated the following functional inequality:

$$
\left\|\sum_{i=1}^{N} f\left(x_{i}\right)\right\| \leq\left\|K f\left(\frac{\sum_{i=1}^{N}\left(x_{i}\right)}{K}\right)\right\| \quad(0<|K| \leq N)
$$

in Fréchet spaces.

In this paper, we investigate the following functional inequalities:

$$
\begin{array}{ll}
\|f(x)+f(y)+f(z)\| \leq\left\|K f\left(\frac{x+y+z}{K}\right)\right\| & (0<|K|<3), \\
\|f(x)+f(y)+K f(z)\| \leq\left\|K f\left(\frac{x+y}{K}+z\right)\right\| & (0<K \neq 2)
\end{array}
$$

and prove the Hyers-Ulam stability of functional inequalities (1.3) and (1.4) in Banach spaces.

Throughout this paper, assume that $X$ is a normed vector space and that $(Y,\|\cdot\|)$ is a Banach space.

\section{Hyers-Ulam stability of functional inequality (1.3)}

Throughout this section, assume that $K$ is a real number with $0<|K|<3$.

Proposition 2.1 Let $f: X \rightarrow Y$ be a mapping such that

$$
\|f(x)+f(y)+f(z)\| \leq\left\|K f\left(\frac{x+y+z}{K}\right)\right\|
$$

for all $x, y, z \in X$. Then the mapping $f: X \rightarrow Y$ is additive.

Proof Letting $x=y=z=0$ in (2.1), we get

$$
\|3 f(0)\| \leq\|K f(0)\|
$$

So, $f(0)=0$.

Letting $z=0$ and $y=-x$ in (2.1), we get

$$
\|f(x)+f(-x)\| \leq\|K f(0)\|=0
$$

for all $x \in X$. So, $f(-x)=-f(x)$ for all $x \in X$.

Letting $z=-x-y$ in (2.1), we get

$$
\|f(x)+f(y)-f(x+y)\|=\|f(x)+f(y)+f(-x-y)\| \leq\|K f(0)\|=0
$$

for all $x, y \in X$. Thus,

$$
f(x+y)=f(x)+f(y)
$$

for all $x, y \in X$, as desired. 
Theorem 2.2 Assume that a mapping $f: X \rightarrow Y$ satisfies the inequality

$$
\|f(x)+f(y)+f(z)\| \leq\left\|K f\left(\frac{x+y+z}{K}\right)\right\|+\phi(x, y, z)
$$

where $\phi: X^{3} \rightarrow[0, \infty)$ satisfies

$$
\widetilde{\phi}(x, y, z):=\sum_{j=1}^{\infty} 2^{j} \phi\left(\frac{x}{2^{j}}, \frac{y}{2^{j}}, \frac{z}{2^{j}}\right)<\infty
$$

for all $x, y, z \in X$. Then there exists a unique additive mapping $A: X \rightarrow Y$ such that

$$
\|A(x)-f(x)\| \leq \frac{1}{2} \widetilde{\phi}(-x,-x, 2 x)+\widetilde{\phi}(x,-x, 0)
$$

for all $x \in X$.

Proof It follows from (2.3) that $\phi(0,0,0)=0$. Letting $x=y=z=0$ in (2.2), we get $\|3 f(0)\| \leq$ $\|K f(0)\|+\phi(0,0,0)=\|K f(0)\|$. So, $f(0)=0$.

Letting $y=x, z=-2 x$ in (2.2), we get

$$
\|2 f(x)+f(-2 x)\| \leq \phi(x, x,-2 x)
$$

for all $x \in X$. So,

$$
\left\|2 f\left(\frac{x}{2}\right)+f(-x)\right\| \leq \phi\left(\frac{x}{2}, \frac{x}{2},-x\right)
$$

for all $x \in X$.

Letting $y=-x$ and $z=0$ in (2.2), we get

$$
\|f(x)+f(-x)\| \leq \phi(x,-x, 0)
$$

for all $x \in X$. It follows from (2.5) and (2.6) that

$$
\begin{aligned}
\left\|2^{l} f\left(\frac{x}{2^{l}}\right)-2^{m} f\left(\frac{x}{2^{m}}\right)\right\| \\
\quad \leq \sum_{j=l}^{m-1}\left\|2^{j} f\left(\frac{x}{2^{j}}\right)-2^{j+1} f\left(\frac{x}{2^{j+1}}\right)\right\| \\
\quad \leq \sum_{j=l}^{m-1}\left\|2^{j} f\left(\frac{x}{2^{j}}\right)+2^{j+1} f\left(\frac{-x}{2^{j+1}}\right)-2^{j+1} f\left(\frac{-x}{2^{j+1}}\right)-2^{j+1} f\left(\frac{x}{2^{j+1}}\right)\right\| \\
\quad \leq \sum_{j=l}^{m-1}\left[\left\|2^{j} f\left(\frac{x}{2^{j}}\right)+2^{j+1} f\left(\frac{-x}{2^{j+1}}\right)\right\|+\left\|2^{j+1} f\left(\frac{-x}{2^{j+1}}\right)+2^{j+1} f\left(\frac{x}{2^{j+1}}\right)\right\|\right] \\
\quad \leq \sum_{j=l}^{m-1} \frac{1}{2} 2^{j+1} \phi\left(\frac{-x}{2^{j+1}}, \frac{-x}{2^{j+1}}, \frac{2 x}{2^{j+1}}\right)+\sum_{j=l}^{m-1} 2^{j+1} \phi\left(\frac{x}{2^{j+1}}, \frac{-x}{2^{j+1}}, 0\right)
\end{aligned}
$$


for all nonnegative integers $m$ and $l$ with $m>l$ and all $x \in X$. It means that the sequence $\left\{2^{n} f\left(\frac{x}{2^{n}}\right)\right\}$ is a Cauchy sequence for all $x \in X$. Since $Y$ is complete, the sequence $\left\{2^{n} f\left(\frac{x}{2^{n}}\right)\right\}$ converges. We define the mapping $A: X \rightarrow Y$ by $A(x)=\lim _{n \rightarrow \infty} 2^{n} f\left(\frac{x}{2^{n}}\right)$ for all $x \in X$. Moreover, letting $l=0$ and passing the limit $m \rightarrow \infty$, we get (2.4).

Next, we show that $A(x)$ is an additive mapping.

$$
\begin{aligned}
\|A(x)+A(-x)\| & =\lim _{n \rightarrow \infty} 2^{n}\left\|f\left(\frac{x}{2^{n}}\right)+f\left(\frac{-x}{2^{n}}\right)\right\| \\
& \leq \lim _{n \rightarrow \infty} \frac{1}{2} 2^{n+1} \phi\left(\frac{2 x}{2^{n+1}}, \frac{-2 x}{2^{n+1}}, 0\right)=0
\end{aligned}
$$

and so $A(-x)=-A(x)$ for all $x \in X$.

$$
\begin{aligned}
\|A(x)+A(y)-A(x+y)\| & =\|A(x)+A(y)+A(-x-y)\| \\
& =\lim _{n \rightarrow \infty} 2^{n}\left\|f\left(\frac{x}{2^{n}}\right)+f\left(\frac{y}{2^{n}}\right)+f\left(\frac{-x-y}{2^{n}}\right)\right\| \\
& \leq \lim _{n \rightarrow \infty} \frac{1}{2} 2^{n+1} \phi\left(\frac{2 x}{2^{n+1}}, \frac{2 y}{2^{n+1}}, \frac{2(x+y)}{2^{n+1}}\right)=0
\end{aligned}
$$

for all $x, y \in X$. Thus, the mapping $A: X \rightarrow Y$ is additive.

Now, we prove the uniqueness of $A$. Assume that $T: X \rightarrow Y$ is another additive mapping satisfying (2.4). Then we obtain

$$
\begin{aligned}
\|A(x)-T(x)\| & =\lim _{n \rightarrow \infty} 2^{n}\left\|A\left(\frac{x}{2^{n}}\right)-T\left(\frac{x}{2^{n}}\right)\right\| \\
& \leq \lim _{n \rightarrow \infty} 2^{n}\left[\left\|A\left(\frac{x}{2^{n}}\right)-f\left(\frac{x}{2^{n}}\right)\right\|+\left\|T\left(\frac{x}{2^{n}}\right)-f\left(\frac{x}{2^{n}}\right)\right\|\right] \\
& \leq \lim _{n \rightarrow \infty}\left[\widetilde{\phi}\left(\frac{x}{2^{n}}, \frac{-x}{2^{n}}, \frac{2 x}{2^{n}}\right)+2 \widetilde{\phi}\left(\frac{x}{2^{n}}, \frac{-x}{2^{n}}, 0\right)\right]=0
\end{aligned}
$$

for all $x \in X$. Then we can conclude that $A(x)=T(x)$ for all $x \in X$. This completes the proof.

Corollary 2.3 Let $p$ and $\theta$ be positive real numbers with $p>1$. Let $f: X \rightarrow Y$ be a mapping satisfying

$$
\|f(x)+f(y)+f(z)\| \leq\left\|K f\left(\frac{x+y+z}{K}\right)\right\|+\theta\left(\|x\|^{p}+\|y\|^{p}+\|z\|^{p}\right)
$$

for all $x, y, z \in X$. Then there exists a unique additive mapping $A: X \rightarrow Y$ such that

$$
\|f(x)-A(x)\| \leq \frac{2^{p}+6}{2^{p}-2} \theta\|x\|^{p}
$$

for all $x \in X$.

\section{Hyers-Ulam stability of functional inequality (1.4)}

Throughout this section, assume that $K$ is a real number with $0<K \neq 2$. 
Proposition 3.1 Let $f: X \rightarrow Y$ be a mapping such that

$$
\|f(x)+f(y)+K f(z)\| \leq\left\|K f\left(\frac{x+y}{K}+z\right)\right\|
$$

for all $x, y, z \in X$. Then the mapping $f: X \rightarrow Y$ is additive.

Proof Letting $x=y=z=0$ in (3.1), we get

$$
\|(K+2) f(0)\| \leq\|K f(0)\| .
$$

So, $f(0)=0$.

Letting $z=0$ and $y=-x$ in (3.1), we get

$$
\|f(x)+f(-x)\| \leq\|K f(0)\|=0
$$

for all $x \in X$. So, $f(-x)=-f(x)$ for all $x \in X$.

Letting $z=\frac{-x-y}{K}$ in (3.1), we get

$$
\left\|f(x)+f(y)-K f\left(\frac{x+y}{K}\right)\right\|=\left\|f(x)+f(y)+K f\left(\frac{-x-y}{K}\right)\right\| \leq\|K f(0)\|=0
$$

for all $x, y \in X$. Thus,

$$
K f\left(\frac{x+y}{K}\right)=f(x)+f(y)
$$

for all $x, y \in X$. Letting $y=0$ in (3.2), we get $K f\left(\frac{x}{K}\right)=f(x)$ for all $x \in X$. So,

$$
f(x+y)=K f\left(\frac{x+y}{K}\right)=f(x)+f(y)
$$

for all $x, y \in X$, as desired.

Theorem 3.2 Let $K$ be a positive real number with $K<2$. Assume that a mapping $f: X \rightarrow$ $Y$ satisfies the inequality

$$
\|f(x)+f(y)+K f(z)\| \leq\left\|K f\left(\frac{x+y}{K}+z\right)\right\|+\phi(x, y, z),
$$

where $\phi: X^{3} \rightarrow[0, \infty)$ satisfies

$$
\widetilde{\phi}(x, y, z):=\sum_{j=1}^{\infty}\left(\frac{2}{K}\right)^{j} \phi\left(\left(\frac{K}{2}\right)^{j} x,\left(\frac{K}{2}\right)^{j} y,\left(\frac{K}{2}\right)^{j} z\right)<\infty
$$

for all $x, y, z \in X$. Then there exists a unique additive mapping $A: X \rightarrow Y$ such that

$$
\|A(x)-f(x)\| \leq \frac{1}{2} \widetilde{\phi}\left(-x,-x, \frac{2}{K} x\right)+\widetilde{\phi}(x,-x, 0)
$$

for all $x \in X$. 
Proof It follows from (3.4) that $\phi(0,0,0)=0$. Letting $x=y=z=0$ in (3.3), we get $\|(K+2) f(0)\| \leq\|K f(0)\|+\phi(0,0,0)=\|K f(0)\|$. So, $f(0)=0$.

Letting $y=-x, z=0$ in (3.3), we get

$$
\|f(x)+f(-x)\| \leq \phi(x,-x, 0)
$$

for all $x \in X$. Letting $x=y=K x, z=-2 x$ in (3.3), we obtain

$$
\|f(K x)+f(K x)+f(-2 x)\| \leq \phi(K x, K x,-2 x)
$$

for all $x \in X$. So,

$$
\left\|\frac{2}{K} f\left(\frac{K}{2} x\right)+f(-x)\right\| \leq \frac{1}{K} \phi\left(\frac{K x}{2}, \frac{K x}{2},-x\right)
$$

for all $x \in X$. It follows from (3.6) and (3.7) that

$$
\begin{aligned}
& \left\|\left(\frac{2}{K}\right)^{l} f\left(\left(\frac{K}{2}\right)^{l} x\right)-\left(\frac{2}{K}\right)^{m} f\left(\left(\frac{K}{2}\right)^{m} x\right)\right\| \\
& \leq \sum_{j=l}^{m-1}\left\|\left(\frac{2}{K}\right)^{j} f\left(\left(\frac{K}{2}\right)^{j} x\right)-\left(\frac{2}{K}\right)^{j+1} f\left(\left(\frac{K}{2}\right)^{j+1} x\right)\right\| \\
& \leq \sum_{j=l}^{m-1}\left[\left\|\left(\frac{2}{K}\right)^{j} f\left[\left(\frac{K}{2}\right)^{j} x\right]+\left(\frac{2}{K}\right)^{j+1} f\left[\left(\frac{K}{2}\right)^{j+1}(-x)\right]\right\|\right. \\
& \left.+\left\|\left(\frac{2}{K}\right)^{j+1} f\left[\left(\frac{K}{2}\right)^{j+1}(-x)\right]+\left(\frac{2}{K}\right)^{j+1} f\left[\left(\frac{K}{2}\right)^{j+1} x\right]\right\|\right] \\
& \leq \sum_{j=l}^{m-1}\left[\frac{1}{K}\left(\frac{2}{K}\right)^{j} \phi\left(-\left(\frac{K}{2}\right)^{j+1} x,-\left(\frac{K}{2}\right)^{j+1} x,\left(\frac{K}{2}\right)^{j} x\right)\right. \\
& \left.+\left(\frac{2}{K}\right)^{j+1} \phi\left(\left(\frac{K}{2}\right)^{j+1} x,\left(\frac{K}{2}\right)^{j+1}(-x), 0\right)\right]
\end{aligned}
$$

for all nonnegative integers $m$ and $l$ with $m>l$ and all $x \in X$. It means that the sequence $\left\{\left(\frac{2}{K}\right)^{n} f\left(\left(\frac{K}{2}\right)^{n} x\right)\right\}$ is a Cauchy sequence for all $x \in X$. Since $Y$ is complete, the sequence $\left\{\left(\frac{2}{K}\right)^{n} f\left(\left(\frac{K}{2}\right)^{n} x\right)\right\}$ converges. So, we may define the mapping $A: X \rightarrow Y$ by $A(x)=$ $\lim _{n \rightarrow \infty}\left(\left(\frac{2}{K}\right)^{n} f\left(\left(\frac{K}{2}\right)^{n} x\right)\right)$ for all $x \in X$.

Moreover, by letting $l=0$ and passing the limit $m \rightarrow \infty$, we get (3.5).

Next, we claim that $A(x)$ is an additive mapping. It follows from (3.6) that

$$
\begin{aligned}
\|A(x)+A(-x)\| & =\lim _{n \rightarrow \infty}\left(\frac{2}{K}\right)^{n}\left\|f\left(\left(\frac{K}{2}\right)^{n} x\right)+f\left(-\left(\frac{K}{2}\right)^{n} x\right)\right\| \\
& \leq \lim _{n \rightarrow \infty}\left(\frac{2}{K}\right)^{n} \phi\left(\left(\frac{K}{2}\right)^{n} x,-\left(\frac{K}{2}\right)^{n} x, 0\right) \\
& =\lim _{n \rightarrow \infty} \frac{K}{2}\left(\frac{2}{K}\right)^{n+1} \phi\left(\left(\frac{K}{2}\right)^{n+1}\left(\frac{2}{K} x\right),\left(\frac{K}{2}\right)^{n+1}\left(-\frac{2}{K} x\right), 0\right) \\
& =0
\end{aligned}
$$

and so $A(-x)=-A(x)$ for all $x \in X$. 
It follows from (3.3) that

$$
\|f(K x)-K f(x)\|=\|f(K x)+f(0)+K f(-x)\| \leq \phi(K x, 0,-x)
$$

for all $x \in X$. Hence,

$$
\begin{aligned}
&\|A(x)+A(y)-A(x+y)\| \\
&=\|A(x)+A(y)+A(-x-y)\| \\
&=\left\|A(x)+A(y)+K A\left(\frac{-x-y}{K}\right)-K A\left(\frac{-x-y}{K}\right)+A(-x-y)\right\| \\
& \leq\left\|A(x)+A(y)+K A\left(\frac{-x-y}{K}\right)\right\|+\left\|A(-x-y)-K A\left(\frac{-x-y}{K}\right)\right\| \\
&=\lim _{n \rightarrow \infty}\left(\frac{2}{K}\right)^{n}\left[\left\|f\left(\left(\frac{K}{2}\right)^{n} x\right)+f\left(\left(\frac{K}{2}\right)^{n} y\right)^{n}+K f\left(\left(\frac{K}{2}\right)^{n} \frac{-x-y}{K}\right)\right\|\right. \\
&\left.\quad+\left\|f\left(\left(\frac{K}{2}\right)^{n}(-x-y)\right)^{n}-K f\left(\left(\frac{K}{2}\right)^{n} \frac{x-y}{K}\right)\right\|\right] \\
& \leq \lim _{n \rightarrow \infty}\left(\frac{2}{K}\right)^{n} \phi\left(\left(\frac{K}{2}\right)^{n} x,\left(\frac{K}{2}\right)^{n} y,\left(\frac{K}{2}\right)^{n} \frac{-x-y}{K}\right) \\
&+\lim _{n \rightarrow \infty}\left(\frac{2}{K}\right)^{n} \phi\left(\left(\frac{K}{2}\right)^{n}(-x-y), 0,\left(\frac{K}{2}\right)^{n} \frac{x+y}{K}\right)=0
\end{aligned}
$$

for all $x, y \in X$. So, the mapping $A: X \rightarrow Y$ is an additive mapping.

Now, we show the uniqueness of $A$. Assume that $T: X \rightarrow Y$ is another additive mapping satisfying (3.5). Then we get

$$
\begin{aligned}
\|A(x)-T(x)\| \\
=\lim _{n \rightarrow \infty}\left(\frac{2}{K}\right)^{n}\left\|A\left(\left(\frac{K}{2}\right)^{n} x\right)-T\left(\left(\frac{K}{2}\right)^{n} x\right)\right\| \\
\leq \lim _{n \rightarrow \infty}\left(\frac{2}{K}\right)^{n}\left[\left\|A\left(\left(\frac{K}{2}\right)^{n} x\right)-f\left(\left(\frac{K}{2}\right)^{n} x\right)\right\|\right. \\
\left.\quad+\left\|T\left(\left(\frac{K}{2}\right)^{n} x\right)-f\left(\left(\frac{K}{2}\right)^{n} x\right)\right\|\right] \\
\leq \lim _{n \rightarrow \infty}\left(\frac{2}{K}\right)^{n} \widetilde{\phi}\left(\left(\frac{K}{2}\right)^{n}(-x),\left(\frac{K}{2}\right)^{n}(-x), \frac{2}{K}\left(\frac{K}{2}\right)^{n} x\right) \\
\quad+2 \lim _{n \rightarrow \infty}\left(\frac{2}{K}\right)^{n} \widetilde{\phi}\left(\left(\frac{K}{2}\right)^{n} x,\left(\frac{K}{2}\right)^{n} x, 0\right)=0
\end{aligned}
$$

for all $x \in X$. Thus, we may conclude that $A(x)=T(x)$ for all $x \in X$. This proves the uniqueness of $A$. So, the mapping $A: X \rightarrow Y$ is a unique additive mapping satisfying (3.5).

Corollary 3.3 Let $p, \theta$ and $K$ be positive real numbers with $p>1$ and $K<2$. Let $f: X \rightarrow Y$ be a mapping satisfying

$$
\|f(x)+f(y)+K f(z)\| \leq\left\|K f\left(\frac{x+y}{K}+z\right)\right\|+\theta\left(\|x\|^{p}+\|y\|^{p}+\|z\|^{p}\right)
$$


for all $x, y, z \in X$. Then there exists a unique additive mapping $A: X \rightarrow Y$ such that

$$
\|f(x)-A(x)\| \leq \frac{\frac{1}{K}\left(\frac{2}{K}\right)^{p}+\frac{6}{K}}{\left(\frac{2}{K}\right)^{p}-\frac{2}{K}} \theta\|x\|^{p}
$$

for all $x \in X$.

Theorem 3.4 Let $K$ be a real number with $K>2$. Assume that a mapping $f: X \rightarrow Y$ satisfies inequality (3.3), where $\phi: X^{3} \rightarrow[0, \infty)$ satisfies

$$
\widetilde{\phi}(x, y, z):=\sum_{j=0}^{\infty}\left(\frac{K}{2}\right)^{j} \phi\left(\left(\frac{2}{K}\right)^{j} x,\left(\frac{2}{K}\right)^{j} y,\left(\frac{2}{K}\right)^{j} z\right)<\infty
$$

for all $x, y, z \in X$. Then there exists a unique additive mapping $A: X \rightarrow Y$ such that

$$
\|A(x)-f(x)\| \leq \frac{1}{2} \widetilde{\phi}\left(x, x,-\frac{2}{K} x\right)+\frac{K}{2} \widetilde{\phi}\left(\frac{2}{K} x,-\frac{2}{K} x, 0\right)
$$

for all $x \in X$.

Proof It follows from (3.9) that $\phi(0,0,0)=0$. Letting $x=y=z=0$ in (3.3), we get $\|(K+2) f(0)\| \leq\|K f(0)\|+\phi(0,0,0)=\|K f(0)\|$. So, $f(0)=0$.

Replacing $x$ by $\frac{2}{K} x$ in (3.7), we get

$$
\left\|f(x)+\frac{K}{2} f\left(-\frac{2}{K} x\right)\right\| \leq \frac{1}{2} \phi\left(x, x,-\frac{2}{K} x\right)
$$

for all $x \in X$. It follows from (3.6) and (3.11) that

$$
\begin{aligned}
& \left\|\left(\frac{K}{2}\right)^{l} f\left(\left(\frac{2}{K}\right)^{l} x\right)-\left(\frac{K}{2}\right)^{m} f\left(\left(\frac{2}{K}\right)^{m} x\right)\right\| \\
& \leq \sum_{j=l}^{m-1}\left\|\left(\frac{K}{2}\right)^{j} f\left(\left(\frac{2}{K}\right)^{j} x\right)-\left(\frac{K}{2}\right)^{j+1} f\left(\left(\frac{2}{K}\right)^{j+1} x\right)\right\| \\
& \leq \sum_{j=l}^{m-1}\left[\left\|\left(\frac{K}{2}\right)^{j} f\left[\left(\frac{2}{K}\right)^{j} x\right]+\left(\frac{K}{2}\right)^{j+1} f\left[\left(\frac{2}{K}\right)^{j+1}(-x)\right]\right\|\right. \\
& \left.+\left\|\left(\frac{K}{2}\right)^{j+1} f\left[\left(\frac{2}{K}\right)^{j+1}(-x)\right]+\left(\frac{K}{2}\right)^{j+1} f\left[\left(\frac{2}{K}\right)^{j+1} x\right]\right\|\right] \\
& \leq \sum_{j=l}^{m-1}\left[\frac{1}{2}\left(\frac{K}{2}\right)^{j} \phi\left(\left(\frac{2}{K}\right)^{j} x,\left(\frac{2}{K}\right)^{j} x,-\left(\frac{2}{K}\right)^{j+1} x\right)\right. \\
& \left.+\left(\frac{K}{2}\right)^{j+1} \phi\left(\left(\frac{2}{K}\right)^{j+1} x,\left(\frac{2}{K}\right)^{j+1}(-x), 0\right)\right]
\end{aligned}
$$

for all nonnegative integers $m$ and $l$ with $m>l$ and all $x \in X$. It means that the sequence $\left\{\left(\frac{K}{2}\right)^{n} f\left(\left(\frac{2}{K}\right)^{n} x\right)\right\}$ is a Cauchy sequence for all $x \in X$. Since $Y$ is complete, the sequence $\left\{\left(\frac{K}{2}\right)^{n} f\left(\left(\frac{2}{K}\right)^{n} x\right)\right\}$ converges. So, we may define the mapping $A: X \rightarrow Y$ by $A(x)=$ $\lim _{n \rightarrow \infty}\left(\left(\frac{K}{2}\right)^{n} f\left(\left(\frac{2}{K}\right)^{n} x\right)\right)$ for all $x \in X$. 
Moreover, by letting $l=0$ and passing the limit $m \rightarrow \infty$, we get (3.10).

The rest of the proof is similar to the proof of Theorem 3.2.

Corollary 3.5 Let $p, \theta$ and $K$ be positive real numbers with $p>1$ and $K>2$. Let $f: X \rightarrow Y$ be a mapping satisfying (3.8). Then there exists a unique additive mapping $A: X \rightarrow Y$ such that

$$
\|f(x)-A(x)\| \leq \frac{\frac{1}{K}\left(\frac{2}{K}\right)^{p}+\frac{6}{K}}{\frac{2}{K}-\left(\frac{2}{K}\right)^{p}} \theta\|x\|^{p}
$$

for all $x \in X$.

\section{Competing interests}

The authors declare that they have no competing interests.

\section{Authors' contributions}

All authors conceived of the study, participated in its design and coordination, drafted the manuscript, participated in the sequence alignment, and read and approved the final manuscript.

\section{Author details}

${ }^{1}$ Department of Mathematics, School of Science, ShenYang University of Technology, Shenyang, 110178, P.R. China.

${ }^{2}$ Department of Mathematics, Research Institute for Natural Sciences, Hanyang University, Seoul, 133-791, Korea.

\section{Received: 30 December 2011 Accepted: 26 November 2012 Published: 12 December 2012}

\section{References}

1. Ulam, SM: A Collection of the Mathematical Problems. Interscience, New York (1960)

2. Hyers, DH: On the stability of the linear functional equation. Proc. Natl. Acad. Sci. USA 27, $222-224$ (1941)

3. Rassias, TM: On the stability of the linear mapping in Banach spaces. Proc. Am. Math. Soc. 72, 297-300 (1978)

4. Gajda, Z: On stability of additive mappings. Int. J. Math. Math. Sci. 14, 431-434 (1991)

5. Rassias, JM: On approximation of approximately linear mappings by linear mappings. Bull. Sci. Math. 108, 445-446 (1984)

6. Rassias, JM: On approximation of approximately linear mappings by linear mappings. J. Funct. Anal. 46, 126-130 (1982)

7. Rassias, JM: On a new approximation of approximately linear mappings by linear mappings. Discuss. Math. 7, 193-196 (1985)

8. Jung, S: Hyers-Ulam-Rassias Stability of Functional Equations in Mathematical Analysis. Hadronic Press, Palm Harbor (2001)

9. Lu, G, Park, C: Hyers-Ulam stability of additive set-valued functional equations. Appl. Math. Lett. 24, 1312-1316 (2011)

10. Park, C: Homomorphisms between Poisson JC*algebra. Bull. Braz. Math. Soc. 36, 79-97 (2005)

11. Park, C: Hyers-Ulam-Rassias stability of homomorphisms in quasi-Banach algebras. Bull. Sci. Math. 132, 87-96 (2008)

12. Park, C, Cho, YS, Han, M: Functional inequalities associated with Jordan-von Neumann type additive functional equations. J. Inequal. Appl. 2007, Article ID 41820 (2007)

13. Cho, YJ, Park, C, Saadati, R: Functional inequalities in non-Archimedean Banach spaces. Appl. Math. Lett. 23, 1238-1242 (2010)

14. Lu, G, Park, C: Functional inequality in Fréchet spaces. Preprint 